\title{
Un posible retrato de la reina María de Hungría pintado por Velázquez para la Corte de Praga
}

The Queen Mary of Hungary, possible portrait painting by Velázquez for the Prague Court

\section{$\mathrm{M}^{\mathrm{a}}$ del Mar Doval Trueba \\ Doctora en Historia del Arte}

Resumen: En la Galería Nacional de Praga (República Checa) se conserva un retrato de la Infanta María de España, hermana de Felipe IV y reina de Hungría y de Bohemia. Conocido sólo por reproducciones de baja calidad, el cuadro ha sido atribuido por ello al taller de Velázquez. Sin embargo, un estudio al natural del mismo, unido a los documentos que sobre esa reina han ido apareciendo, han permitido proponer la obra como salida del pincel de Diego Velázquez, pintor de Cámara de Felipe IV.

Palabras clave: María de Hungría, Velázquez, retrato, Praga, siglo XVII, Corte.

Abstract: A portrait of the Infanta María of Spain, Phillip IV's sister and queen of Hungary and Bohemia is conserved in the Czech Republic belonging to the National Gallery of Prague.

So far the picture has been known only for low-quality reproductions and attributed, therefore, to Velázquez's workshop.

Nevertheless, an analysis to the natural one joint to the documents discovered about this queen, has permitted to us consider it, probably, as a work out of the direct brush of Diego Velázquez, Phillip IV's court painter.

Keywords: Mary of Hungary, Velázquez, portrait, Prague, $17^{\text {th }}$ century, Court. 


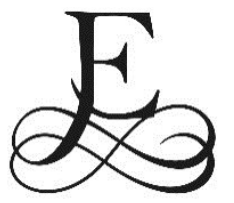

I Museo del Prado conserva un retrato de la Infanta María de Hungría de manos de Diego Velázquez (L. 0,58 x 0,44,

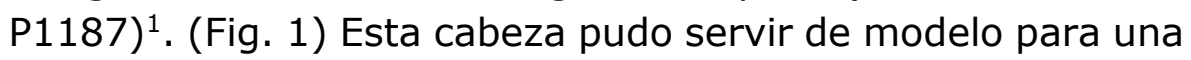
serie de lienzos los cuales se reparten por varios museos de distintos países. El estudio comparativo, atendiendo a criterios cualitativos, nos ha permitido ir diferenciando unos de otros, y nos ha llevado a primar el retrato de cuerpo entero perteneciente a la Galería Nacional (Narodni Gallery) de Praga, (Fig. 2) como salido de la mano del pintor de Cámara de Felipe IV.

El 25 de abril de 1629 la Infanta María, hija de Felipe III y de Margarita de Austria, y hermana de Felipe IV, contraía matrimonio por poderes, en Madrid, con su primo Fernando, rey de Hungría y de Bohemia, y futuro emperador al suceder en el trono a su padre Fernando II. La comitiva partió de Madrid el 26 de diciembre de 1629; la reina fue escoltada por sus tres hermanos hasta Zaragoza: Felipe IV (1605-1665), el Infante don Carlos (1607-1632) y el Infante don Fernando (1609-1641).

Tras la marcha de los hermanos, se puso al frente de la comitiva, por encargo del propio Felipe IV, don Antonio Álvarez de Toledo y Beaumont, V Duque de Alba (1568-1639). Es el propio Duque quien narra las peripecias del viaje al Conde-Duque de Olivares en carta conservada en el Archivo de la Casa de Alba, ya publicada en trabajos anteriores ${ }^{2}$. La jornada no iba a estar exenta de problemas y es que, al llegar a Italia se encontraron con una epidemia de peste que les impidió realizar el trayecto deseado ${ }^{3}$. Por tierra no se podía llegar a Trento, lugar elegido por el propio Emperador para el encuentro; otra opción era la travesía del río Po hasta Ferrara, pero necesitaban embarcaciones suficientemente grandes para no tocar la ribera, por miedo al contagio de los lugareños. Tras permanecer un mes en Génova, optaron por el camino más complicado que era marchar a Nápoles, donde llegaron en agosto por vía marítima, la opción menos preferida de don Antonio, dado los vientos con los que se iban a encontrar durante la travesía, pero la más conocida al haber sido él, anteriormente, Virrey ${ }^{4}$.

\footnotetext{
${ }^{1}$ Charles B. Curtis, Velázquez y Murillo, (New York: J.W. bouton, 1883), p. 244; J. Allende-Salazar, (ed.), Velázquez. Des Meisters Gemälde. Por Walter Gensel, (Berlin y Leipzig: 1925), no 45; A. L. Mayer, Velázquez: A catalogue raisonné of the pictures and drawings, (Londres: Faber \& Faber,1936), no 507; E. Lafuente Ferrari, Velázquez. Complete edition, (Londres: Phaidon Press, 1943), no 50; E. Lafuente, Velázquez. Edición completa, (Barcelona: Ediciones selectas, 1944), no 43; Bernardino de Pantorba, La vida y obra de Velázquez, (Madrid: Compañía Bibliográfica Española, 1955), no 40; J. López-Rey, Velázquez: A catalogue raisonné of his oeuvre, (Londres: Faber \& Faber, 1963), no 379; P. M. Bardi, La obra pictórica completa de Velázquez, (Barcelona: Noguer y Rizzoli Editores, 1970, 1973, 1982), no 42; J. Gudiol, Velázquez, (Barcelona: Ed. Polígrafa, 1973), no 46; P. Ŝtěpánek, "Nuevas pinturas españolas de Praga" Ibero-Americana Pragensia, VIII, (1974), 145-147; E. Harris y J. H. Elliott, "Velázquez and the Queen of Hungary", The Burlington Magazine, 118, (1976), pp. 24-26; J. López-Rey, Velázquez. The artist as a maker, (Lausana-París: Bibliothêque des Arts: 1979), no 48; J. Brown, Velázquez. Pintor y cortesano, (Madrid: Alianza Editorial, 1986), no 53; Julián Gállego, "Catálogo de pinturas", Velázquez, dir. Alfonso E. Pérez Sánchez, (Madrid: Museo del Prado, 1990), no 22, J. Portús, Velázquez. Guía, Madrid: Museo del Prado, 1999), p. 76.

${ }^{2}$ Archivo de Alba (ADA), C. 82- no 37, 2v. El viaje completo se estudia en M. del Mar Doval Trueba, "Sobre dos viajes a Italia: el de María de Hungría y el primero de Velázquez", Boletín del Museo e Instituto Camón Aznar, no 105, (2010), pp. 69-96.

${ }^{3}$ Para más información sobre dicho viaje véase Doval Trueba, "Sobre dos viajes", pp. 69-96.

${ }^{4}$ En la Colección del Banco de Santander se conserva un cuadro de Domenico Gargiulo, Micco Spadaro que se ha venido considerando la entrada de la comitiva regia en Nápoles, "Desembarco de la infanta María de Austria en Nápoles (L. 200 × 310 cm. Madrid, Fundación Banco de Santander). Recientemente,
} 


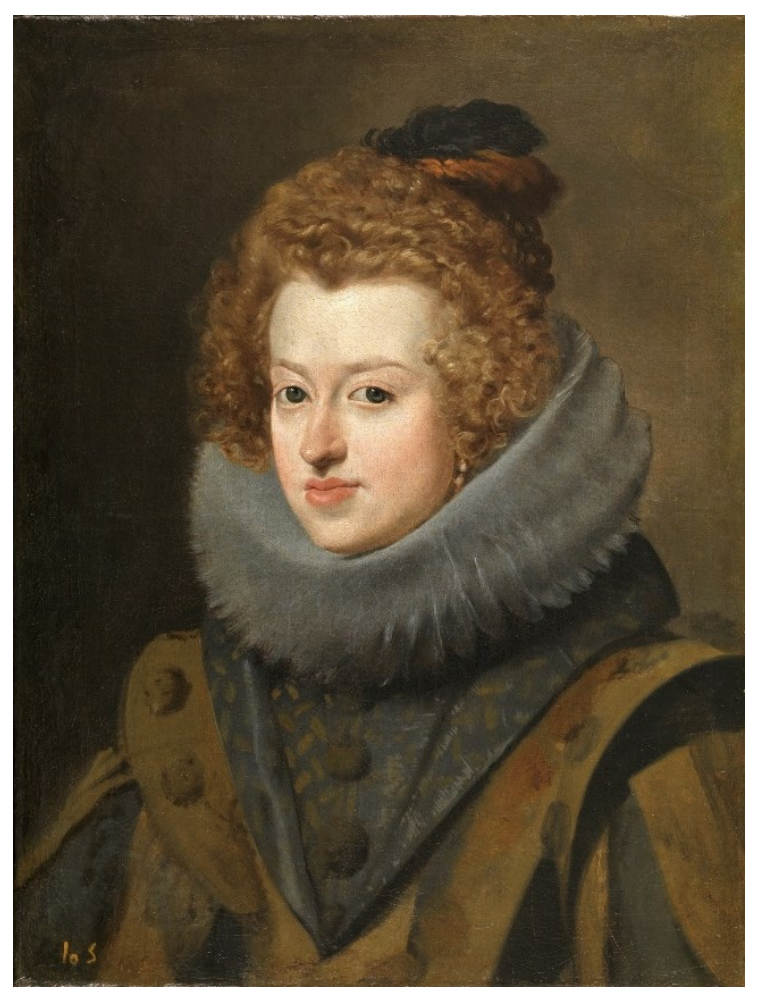

Fig. 1. Diego Velázquez, Doña María de Austria. Reina de Hungria. Madrid, Museo Nacional del Prado

C) (inv. P01187)

Para la salida, tras muchas deliberaciones y, ante las amenazas de los turcos a Venecia, la necesidad de galeras para defenderse, la continuidad de la peste y que el invierno era duro y no podrían circular los carros de bueyes, se eligió un itinerario a propuesta del Conde de Franquenburg (Frankenberg ${ }^{5}$ ), embajador imperial: Pescara, Ascoli, Fermo, Loreto, Ancona, Senigallia, Fano, Pésaro, Rímini, Ferrara, Treviso y Trento punto último e inamovible, por voluntad expresa del Emperador, y previa una cuarentena de dos meses antes de entrar en los dominios imperiales ${ }^{6}$.

Teresa Zapata Fernández de la Hoz considera que, en realidad, el cuadro representa la entrada de la hija de ésta, Mariana de Austria, cuando vino a España para contraer matrimonio con Felipe IV. Teresa Zapata Fernández de la Hoz, La corte de Felipe IV se viste de fiesta: la entrada de la reina Mariana de Austria, (Universidad de Valencia, 2016), Fig. 16.

Más bibliografía sobre Micco Spadaro: Paintings by Old Masters, (Londres: Somerville \& Simpson Ltd, 1975). Cat. venta. Reino Unido. Londres: Giancarlo Sestieri, Domenico Gargiulo Detto Micco Spadaro. Paesaggista e Cronista Napolitano, (Milano, Roma: Jandi Sapi Editori, 1994); Micco Spadaro. Napoli Ai Tempi Di Masaniello, (Nápoles: Electa, 2002), cat. Exp.

${ }^{5}$ Sobre más documentación relacionada con el Conde de Frankenberg, embajador imperial en Madrid, ver León Pinelo, Anales de Madrid: reinado de Felipe III, años de 1598 a 1621, ed. y estudio crítico del manuscrito no 1255 de la Biblioteca Nacional por Ricardo Martorell Téllez-Girón, (Madrid: Estanislao Maestre, 1931). En su página 249 hace referencia al año 1929 y al parecer del conde de Frankenberg sobre el viaje que estamos analizando (Archivo de Simancas, Estado, Legajo 2510) Año 1629.

${ }^{6}$ ADA, C. 20, no 137,139,141 en Doval Trueba, 2010, p. 79. 


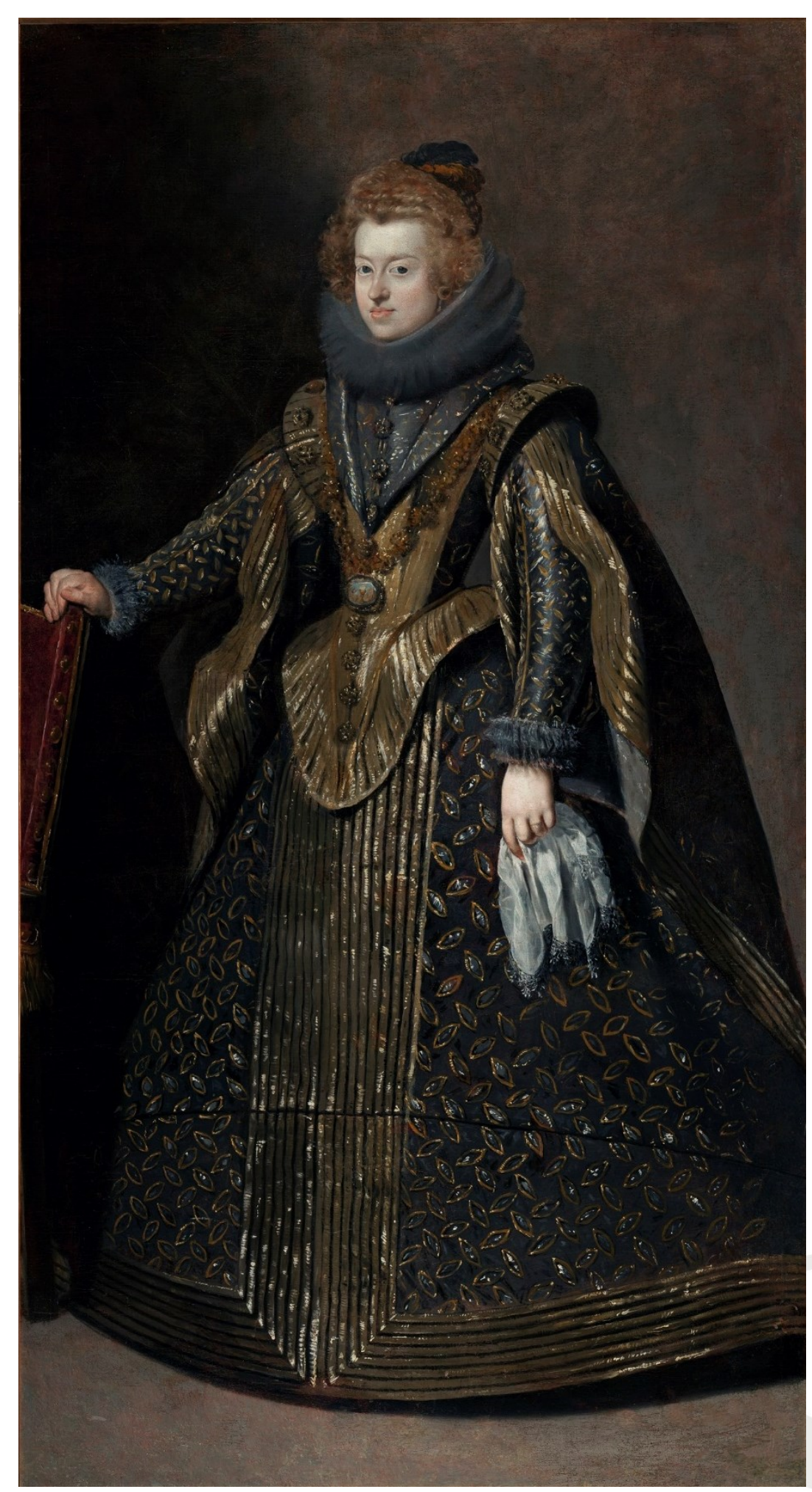

Fig. 2. Taller de Diego Velázquez (¿), Doña María de Hungría. Praga, Narodni Gallery®

Algunos de los lugares mencionados se incluyeron, pero en sentido opuesto, en el recorrido de otro viaje cuyo punto de partida fue el Alcázar de Madrid, mucho más discreto, aunque no exento de importancia, al menos desde una perspectiva artística: el que iniciara Diego Velázquez por esas mismas fechas hasta llegar a coincidir con la futura emperatriz en Nápoles. 
Francisco Pacheco, suegro del pintor, nos cuenta que "fue a parar a Venecia"7. Pasó por Cento, por Loreto y en Bolonia "no paró ni a dar cartas al Cardenal Ludovico ni al Cardenal Espada"8. En Roma permaneció un año y, parafraseando nuevamente a Pacheco, "determinose de volver a España, por la mucha falta que hacía, y a la vuelta paró en Nápoles, donde pintó un lindo retrato de la Reina de Hungría para traerlo a Su Magestad"9. Una vez realizado el cometido y aprovechando las galeras que regresaban a España con los criados que habían acompañado a la reina hasta Nápoles, volvió él mismo en ellas con un criado, según consta en documentos conservados en el Archivo de Palacio de Madrid ${ }^{10}$.

Zarco del Valle identificó el cuadro mencionado más arriba con un retrato registrado en el inventario de los bienes recogidos en las habitaciones de trabajo de Velázquez en el Alcázar, realizado por el nuevo Aposentador Francisco de Contreras, por diligencia de Martínez del Mazo y Gaspar de Fuensalida, amigo del pintor, y por orden del propio Felipe IV, el 10 de agosto de 1660: un Retrato de la Sra. Infanta Reyna de ungria ${ }^{11}$. Fue Madrazo quien, en su catálogo extenso del Museo del Prado lo relacionó con el "Retrato de Doña María de Austria, reina de Hungría" conservado en el Museo del Prado, que venimos estudiando el cual se encontraba, en 1794, en la Quinta del Duque del Arco ( $n^{\circ} 135$ ) y en 1808 en el Palacio del Buen Retiro.

La infanta lleva lechuguilla de gasa agrisada sobre un vestido leonado con cuello abierto en uve por delante y levantado por detrás. Tanto en el escote como en los brahones del traje, aparece una decoración de botones o borlas ${ }^{12}$.

A pesar de que Enriqueta Harris y Elliott consideran que forma parte de un conjunto de retratos de miembros de la Familia Real encargados a Velázquez en $1628^{13}$, y en ese mismo sentido se manifiesta McKim-Smith ${ }^{14}$, López-Rey y Brown lo datan en fecha posterior.

Bernardino de Pantorba (1955) ${ }^{15}$ y Julián Gállego apuntan la posibilidad de que esta cabeza del Prado fuera un boceto para un cuadro de mayores dimensiones. Para el último autor, el retrato mencionado no se llegó a realizar; a su vez, piensa no debió de ser muy apreciado por el rey al haberlo

\footnotetext{
${ }^{7}$ Ver Francisco Pacheco, El Arte de la Pintura, ed. Revisada por Bonaventura Bassegoda, (Madrid: Cátedra, 1990), pp. 206-209.

8 Pacheco, Arte Pintura, pp. 207-208.

${ }^{9}$ Íbidem, p. 209.

${ }^{10}$ Archivo General de Palacio (AGP), Sección Histórica, Caja 193. La relación aparece por triplicado. En Doval Trueba, 2010, p. 80.

${ }_{11}$ M. M. Zarco del Valle, Documentos inéditos para la historia de las Bellas Artes en España, (Madrid: Imprenta de la Viuda de Calero, 1876), p. 222.

12 Sobre la indumentaria de ese momento: Miguel Herrero, "Estudio de indumentaria española de los siglos XVI y XVII" en Hispania: Revista española de historia, No 19, (1945), págs. 286-307; Fernando Marcos Álvarez, Algunas precisiones léxicas sobre indumentaria española en el siglo XVI, Actas del II Congreso Internacional de Historia de la Lengua española, coord. por Manuel Ariza Viguera, Vol. 1, (1992), págs. 1161-1172; Margarita Tejeda Fernández, "Léxico de indumentaria regia y cortesana en España: siglos XVII y XVIII" en Boletín de arte, No 26-27, (2005-2006), págs. 865-866; Miguel Herrero Garcia, Estudios sobre indumentaria española en la época de los Austrias, (Madrid: Centro de Estudios Europa Hispánica, 2014).

13 Harris y Elliott, "Velázquez and the Queen", pp. 24-26.

${ }^{14}$ G. McKim-Smith, y R. Newman, Ciencia e historia del Arte. Velázquez en el Prado, (Madrid: Museo del Prado, 1993), p. 57, il. 32.

${ }^{15}$ B. Pantorba, La vida y la obra de Velázquez, (Madrid: Compañía bibliográfica española, 1955), p.40.
} 
dejado olvidado en el taller del pintor $^{16}$. Por su parte Gudiol y Brown lo consideran un modelo para uso del taller ${ }^{17}$.

En realidad, al tratarse de un estudio de una cabeza, el cuadro se encontraba en el lugar apropiado para ser utilizado como modelo en varias de las versiones existentes. Francisco Pacheco insiste en la realización de bocetos previos al retrato individual $y$, especialmente, la ejecución de cabezas. Sobre su yerno recoge un suceso de juventud relacionada con su ejercicio en este campo. De todos es conocido el siguiente comentario:

"Con esta doctrina [dibujos de cabezas] se crió mi yerno, Diego Velásquez de Silva, siendo muchacho, el cual tenía cohechado un aldeanillo aprendiz, que le servía como modelo en diversas acciones y posturas, ya llorando, ya riendo, sin perdonar dificultad alguna. Y hizo por él muchas cabezas de carbón y realce, en papel azul y de otros muchos naturales, con que granjeó la certeza en el retratar"18.

Famosa es la anécdota relatada por Palomino relacionada con el pintor de Cámara: "Era muy agudo en sus dichos y respuestas: díjole un día Su Majestad, que no faltaba, quien dijese, que toda su habilidad se reducía a saber pintar una cabeza; a que respondió: Señor, mucho me favorecen porque yo no sé, que haya quien la sepa pintar"19.

Volviendo a Pacheco en su tratado sobre El Arte de la Pintura, nos desvela su propio método de trabajo basado en la realización continua de bocetos (la "elaboración de numerosos bocetos es la mejor garantía para poder elegir de entre ellos el más ajustado y perfecto"20). Su yerno quien, "sigue este camino, también se ve la diferencia que hace a los demás, por tener siempre delante el natural"21.

Las enseñanzas de Pacheco se plasman en un riguroso método de trabajo del cual tenemos varios ejemplos, ya analizados en trabajos anteriores ${ }^{22}$. Uno de ellos son los retratos de Isabel de Borbón. En el Monasterio de El Escorial se conserva una cabeza de esta reina (L. 69,5 x 58, 5 cm. Inv. no 10014149) la cual pudo servir como modelo al de Copenhague de cuerpo entero (L. 218 x $127,5 \mathrm{~cm}$. Inv. KMSsp687), Gudiol recoge otro retrato de similares características en la Colección Frascione de Florencia ${ }^{23}$.

En el caso de la reina de Hungría, la cabeza del Museo del Prado, de muy parecidas dimensiones a la mencionada de su cuñada, pudo también servir como modelo al resto de versiones de cuerpo entero.

\footnotetext{
16 J. Gállego, Velázquez, Cat. Exp. (Museo del Prado: Madrid, 1990) p. 154

17 Brown, Velazquez pintor y cortesano, (Madrid: Alianza editorial, 1986).; Enriqueta Harris, Velázquez, (Vitoria-Gasteiz: Ephialte, 1991), p. 70, il.nº 61.

18 Pacheco, Arte Pintura, p. 528.

${ }^{19}$ Ver A. A. Palomino, El museo pictórico y la escala óptica: práctica de la pintura, en que se trata el modo de pintar a el Óleo, Temple... (1796-1797), (Madrid: Imprenta de Sancha, 1795), T. III.

20 Íbidem, p. 433.

${ }^{21}$ Ídem

22 Ma del Mar Doval Trueba, "Velázquez y los retratos de Isabel de Borbón", Boletín del Museo e Instituto Camón Aznar, no 103, (2009), p. 177 y ss.

${ }^{23}$ Doval Trueba, "Velázquez y los retratos", p. 144, nota 21
} 
Volviendo a María de Hungría, se conservan varios retratos de cuerpo entero, los cuales serán objeto de estudio en un trabajo posterior, en la Gemäldegalerie de Berlín (L. $208 \times 109$ cm. Inv. no 413c); en la Hispanic Society of America (L. 204 x 175 cm.); colección Fluxá en Madrid (L. 210 x $118 \mathrm{~cm}$.) con una inscripción: "Egurdiriksen Ft. 1630". Por otro lado, en el inventario del Marqués de Leganés de 1642 se registra un retrato de La reyna de Ungria, [de la mano de Velázquez] ${ }^{24}$.

Por último, otra conservada en Praga, como depósito de la Narodni Galerie en el castillo Decin (L. 201 x 108 cm. inv. DO 4984), es la que presenta una mayor calidad (Fig. 2). En cuanto a la procedencia del cuadro del Conde de Thun (Bohemia) en 1646, año que coincide con el del fallecimiento de la reina y ha permanecido en dicha colección hasta 1946, fecha en la que ingresó en la Galería Nacional de Praga como bien incautado.

Doña María se nos presenta de cuerpo entero, con un pañuelo en su mano izquierda y la derecha apoyada sobre el respaldo de un sillón. Como es propio del pintor en esta etapa, prescinde de cortinaje. Tanto el cabello como la lechuguilla están tratados con la manera suelta fácilmente reconocible en su pincel. El vestido, los encajes del pañuelo y las mangas, los brillos del hilo de plata responden a un toque virtuoso de alguien muy diestro en su oficio. A diferencia de la cabeza de Madrid, aquí el vestido de la reina se adorna con un gran collar en cuyo medallón, situado a la altura de la cintura, se recogen motivos eucarísticos.

José López-Rey no incluye este lienzo en su primer catálogo sobre Velázquez donde recoge el resto de variaciones existentes conocidas por él ${ }^{25}$. Sí aparece ya en la edición de 1996 del Widenstein Institute supervisada y revisada por Odile Delenda ${ }^{26}$. La reproducción, en blanco y negro, de escasa calidad, impide apreciar la maestría del toque en la pintura. Por su parte, Pavel Stepanek la considera obra de Velázquez con participación de su taller, hacia $1628-1630^{27}$.

El pintor de Cámara, en su primera etapa al servicio del monarca, no pudo evitar enfrentarse a esos vestidos con bordados tan minuciosos, tan alejados de su manera posterior ya esbozada en parte de esta obra28. De hecho,

\footnotetext{
24 M. Crawford Volk., "New Light on a Seventeenth-Century Collector: The Marquis of Leganes", Art Bulletin, 52, (1980), p. 268.; J.J. Pérez Preciado, El marqués de Leganés y las aartes. Tesis doctoral, (Madrid: UCM, 2010), p.364, no 496.

${ }^{25}$ López-Rey, Catalogue raisonné, pp. 248- 9, no 379, 380, 381, 382, 383.

26 J. López-Rey, Velázquez. Painter of painters, ed. Revisada por Odile Delenda, Köln, (1996), T.I, p. 81.

27 Pavel Ŝtĕpánek, "Nuevas pinturas españolas de Praga", Ibero-Americana Pragensia, VIII, (1974), pp. 145-147, il.

${ }^{28}$ En este sentido, Jusepe Martínez recoge una graciosa anécdota que le aconteció al pintor de Cámara muy ilustrativa sobre esta manera de pintar: "Por estos sucesos te aconsejaré, estudioso mío, no dés por este camino de retratos, que se sujeta un hombre a oír muchas simplicidades e ignorancias: y aunque parece que esto bastaba para tu desengaño, no obstante, entre los muchos casos que te puedo contar, he escogido este solo para último en esta materia. Estando Diego Velázquez en esta ciudad de Zaragoza, asistiendo a S.M. Don Felipe IV de gloriosa memoria, le pidió un caballero le hiciera un retrato de una hija suya muy querida: hízolo (sic) con tanto gusto que salió con grande escelencia (sic), al fin como de su mano: hecha que fue la cabeza, para lo restante del cuerpo, por no cansar a la dama, lo trajo a mi casa para acabarlo, que era de medio cuerpo: llevólo después acabado a casa del caballero; viéndolo la dama dijo, que por ningún caso había de recibir tal retrato, y preguntándole su padre en que se fundaba, respondió: que en todo no le agradaba, pero en particular que la balona que ella llevaba, cuando la retrató
} 
constituyen el elemento más importante para mostrar el status de la dama en cuestión, en este caso de la futura emperatriz de Austria. Y, por otro lado, resulta impensable que Velázquez dejara la imagen de una persona de tal categoría en manos de su taller; una vez creada la iconografía, si se necesitaban más copias, sí intervendrían sus ayudantes. Por último, tratándose de la reina de Hungría y Bohemia el cuadro se conserva en la capital del que fue su reino, adonde se dirigía.

Aprovechando la estancia del pintor de su propio hermano Felipe IV en Nápoles, el retrato pudo convertirse en un posible regalo entre recién casados que se uniría a todos los realizados con antelación y, desde luego, en la imagen oficial de la nueva reina para sus súbditos. Un retrato, además, en el cual el pintor de Cámara de Felipe IV plasmaría todos los nuevos conocimientos aprendidos en su primer periplo italiano. Finalizado éste, Velázquez regresaría a España con el retrato de pequeñas dimensiones del Prado y el grande marcharía a su destino en la capital de Bohemia.

El cuadro de Madrid está pintado sobre un lienzo reentelado con posterioridad con una tela de densidad del hilo elevada, el bastidor tensado por medio de cuñas ${ }^{29}$. El cuadro de Praga también fue pintado en lienzo, alejándose del mantelillo característico de la pintura veneciana, Tiziano entre otros; está reentelado y el bastidor se tensa de la misma manera que el conservado en la pinacoteca madrileña.

Poco a poco se va configurado un modo de trabajar donde Velázquez, como pintor de cámara de Felipe IV, se coloca al frente de un grupo de artistas cuya calidad y buen hacer fueron nota común en la Europa del siglo XVII.

era de puntas de Flandes muy finas. Paréceme que esto basta para ejemplar". Jusepe Martínez, Discursos practicables del nobilísimo arte de la pintura, edición de Julián Gállego, (Madrid: Akal 1988), pp. 211-212 ${ }^{29}$ Carmen Garrido, Velázquez. Técnica y Evolución, Museo del Madrid, 1992, p. 195 
Fuentes documentales:

Madrid, Archivo de Alba (ADA), C. 82- no 37, 2v.

Madrid, ADA, C. 20, no 137,139,141

Madrid, Archivo General de Palacio (AGP), Sección Histórica, Caja 193.

Simancas, Archivo de Simancas, Estado, Legajo 2510, Año 1929

Bibliografía:

Allende-Salazar 1925: J. Allende-Salazar, (ed.), Velázquez. Des Meisters Gemälde. Por Walter Gensel, (Berlin y Leipzig:1925).

Bardi 1982: P. M. Bardi, La obra pictórica completa de Velázquez, (Barcelona: Noguer y Rizzoli Editores, 1970, 1973, 1982).

Brown 1986: J. Brown, Velázquez. Pintor y cortesano, (Madrid: Alianza Editorial, 1986).

Colomer 2014: J. L. Colomer y Amalia Descalzo, dirs., Vestir a la española en las cortes europeas (Siglos XVI y XVII), (Madrid: CEEH, 2014).

Crawford Volk 1980: M. Crawford Volk., "New Light on a SeventeenthCentury Collector: The Marquis of Leganes", Art Bulletin, 52, (1980), pp. 256268.

Curtis 1883: Charles B. Curtis, Velázquez y Murillo, (New York: J.W. Bouton, 1883)

Doval Trueba 2009: M. M. Doval Trueba, "Velázquez y los retratos de Isabel de Borbón", Boletín del Museo e Instituto Camón Aznar, n 0 103, (2009), pp. 137-157. p. 177 y ss.

Doval Trueba 2010: M. del Mar Doval Trueba, "Sobre dos viajes a Italia: el de María de Hungría y el primero de Velázquez", Boletín del Museo e Instituto Camón Aznar, no 105, (2010), pp. 69-96.

Gállego 1990: Julián Gállego, "Catálogo de pinturas", Velázquez, dir. Alfonso E. Pérez Sánchez, (Madrid: Museo del Prado, 1990).

Gallego 1990: Julián Gállego, Velázquez, Cat. De la Exp. Museo del Prado, 1990.

Garrido 1992: Carmen Garrido, Velázquez. Técnica y Evolución, (Museo del Prado: Madrid, 1992).

Giancarlo 1994: Giancarlo Sestieri, Domenico Gargiulo Detto Micco Spadaro. Paesaggista e Cronista Napolitano, (Milano, Roma: Jandi Sapi Editori, 1994).

Gudiol 1973: J. Gudiol, Velázquez, (Barcelona: Ed. Polígrafa,1973).

Harris 1991: Enriquetta Harris, Velázquez, (Vitoria-Gasteiz: Ephialte, 1991)

Harris y Elliot 1976: E. Harris y J.H. Elliott, "Velázquez and the Queen of Hungary", Burlington Magazine, 118, (1976), pp. 24-26. 
Herrero 1945: Miguel Herrero, "Estudio de indumentaria española de los siglos XVI y XVII" en Hispania: Revista española de historia, no 19, (1945), pp. 286-307.

Herrero García 2014: Miguel Herrero Garcia, Estudios sobre indumentaria española en la época de los Austrias, (Madrid: Centro de Estudios Europa Hispánica, 2014).

Herrero García 2014: Miguel Herrero García, Los tejidos en la España de los Austrias. Fragmentos de un diccionario, (Madrid: CEEH, 2014).

Lafuente Ferrari 1943: E. Lafuente Ferrari, Velázquez. Complete edition, (Londres: Phaidon Press, 1943).

Lafuente Ferrari 1944: E. Lafuente, Velázquez. Edición completa, (Barcelona: Ediciones selectas, 1944).

López-Rey 1963: J. López-Rey, A Catalogue raisonné of his oeuvre, (London: Faber\& Faber, 1963).

López-Rey 1963: J. López-Rey, Velázquez: A catalogue raisonné of his oeuvre, (Londres: Faber \& Faber, 1963).

López-Rey 1979: J. López-Rey, Velázquez. The artist as a maker, (LausanaParís: Bibliothêque des Arts: 1979).

López-Rey 1996: J. López-Rey, Velázquez. Painter of painters, ed. Revisada por Odile Delenda, (Köln, 1996), vol. I.

Marcos Álvarez 1992: Fernando Marcos Álvarez, Algunas precisiones léxicas sobre indumentaria española en el siglo XVI, Actas del II Congreso Internacional de Historia de la Lengua española, coord. por Manuel Ariza Viguera, vol. 1, (1992), págs. 1161-1172.

Martínez ed. Gállego 1988: Jusepe Martínez, Discursos practicables del nobilísimo arte de la pintura, edición de Julián Gállego, (Madrid: Akal, 1988).

Mayer 1936: A. L. Mayer, Velázquez: A catalogue raisonné of the pictures and drawings, (Londres: Faber \& Faber, 1936).

Micco 1975: Micco Spadaro. Paintings by Old Masters, (Londres: Somerville \& Simpson Ltd, 1975).

Micco 2002: Micco Spadaro. Napoli Ai Tempi Di Masaniello, (Nápoles: Electa, 2002), cat. Exp.

McKim-Smith y Newman 1993: G. McKim-Smith, y R. Newman, Ciencia e historia del Arte. Velázquez en el Prado, (Madrid: Museo del Prado, 1993).

Pacheco, ed. Bassegoda 1990: Francisco Pacheco, El Arte de la Pintura, ed. revisada por Bonaventura Bassegoda, (Madrid: Cátedra, 1990).

Palomino 1988: A. A. Palomino, El museo pictórico y la escala óptica: práctica de la pintura, en que se trata el modo de pintar a el Oleo, Temple... (17961797), tomo III, (Madrid: Imprenta de Sancha, 1795). 
Pantorba 1955: Bernardino de Pantorba, La vida y obra de Velázquez, (Madrid: Compañía Bibliográfica Española, 1955).

Pinelo, ed. Martorell Téllez-Girón 1931: León Pinelo, Anales de Madrid: reinado de Felipe III, años de 1598 a 1621, ed. y estudio crítico del manuscrito no 1255 de la Biblioteca Nacional por Ricardo Martorell TéllezGirón, (Madrid: Estanislao Maestre, 1931).

Portús 1999: J. Portús, Velázquez. Guía, (Madrid: Museo del Prado, 1999), p. 76.

Ŝtĕpánek 1974: Pavel Ŝtěpánek, "Nuevas pinturas españolas de Praga" Ibero-Americana Pragensia, VIII, (1974), pp. 145-147.

Tejeda Fernández 2005-2006: Margarita Tejeda Fernández, "Léxico de indumentaria regia y cortesana en España: siglos XVII y XVIII" en Boletín de arte, no 26-27, (2005-2006), pp. 865-866.

Zapata Fernández de la Hoz 2016: La corte de Felipe IV se viste de fiesta: la entrada de la reina Mariana de Austria, (Valencia: Universidad de Valencia, 2016).

Zarco del Valle 1876: M. M. Zarco del Valle, Documentos inéditos para la historia de las Bellas Artes en España, (Madrid: Imprenta de la Viuda de Calero, 1876).

Recibido: 22/09/2017

Aceptado: 2/03/2018 